\title{
Editorial
}

\section{Hearing Difficulties in the Absence of Hearing Loss}

DOI: 10.3766/jaaa.29.6.1

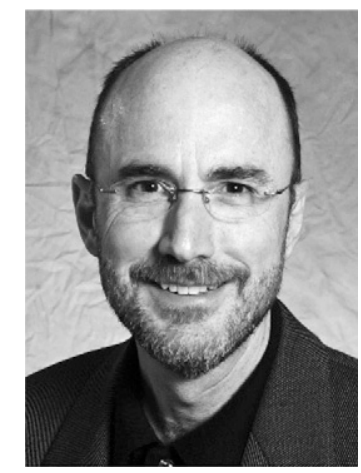

$\mathrm{I}$ tis not an uncommon occurrence to conduct an audiometric assessment on a patient who has been referred for hearing loss and finding normal puretone thresholds and excellent word recognition ability in quiet. However, these same individuals may report difficulties in speech recognition in more challenging environments, for example, where even modest amounts of background noise is present. These individuals may also self-report significant hearing handicap. The finding of hearing difficulties despite normal audiometric pure tone thresholds has been reported in a number of disorders including: traumatic brain injury, and auditory neuropathy spectrum disorder. In this issue of the Journal, Roup and colleagues from The Ohio State University report their observations evaluating 39 subjects that were divided into two groups. One group of 20 subjects had normal audiometric data and did not complain of hearing difficulties. A second group of 19 subjects with normal audiometric data complained of hearing difficulties. The two groups were examined using a number of measures including SCAN: 3-A, dichotic digit recognition, gaps-in-noise test, the masking level difference (MLD) test at 500 $\mathrm{Hz}$ and the speech recognition in noise (SPIN) test. Additionally, both groups completed the Hearing Handicap
Inventory for Adults (HHIA) and the Auditory Processing Questionnaire (APQ).

There was a treatment arm to this study. That is, for the group with hearing difficulties (i.e., despite normal pure tone audiometry) subjects were fit with mild gain amplification that was programmed to provide $5-10 \mathrm{~dB}$ of insertion gain from $1000-4000 \mathrm{~Hz}$. The subjects were asked to wear the hearing aids for at least 4 hours per day. The subjects were re-assessed one month after the fitting.

It should not surprise you that the group with hearing difficulties volunteered significantly greater hearing handicap and auditory processing difficulties than the control group. Further, performance on hearing assessments was poorer for the group with hearing difficulties. What might surprise you is that the experimental group with hearing difficulties showed significant improvement in hearing handicap, auditory processing difficulties, and speech in background noise, compared to the control subjects.

I have glossed-over some of the high points of this significant investigation. The editors hope you, like us, will find this study of great interest.

Gary P. Jacobson, Ph.D. Editor-in-Chief 